\title{
GREEN SYNTHESIS OF COPPER NANOPARTICLE FROM PASSIFLORA FOETIDA LEAF EXTRACT AND ITS ANTIBACTERIAL ACTIVITY
}

\author{
SAMAR FATMA ${ }^{1}$, KALAINILA P ${ }^{1}$, ERNEST RAVINDRAN² ${ }^{2}$ RENGANATHAN $\mathbf{S}^{1 *}$ \\ ${ }^{1}$ Department of Biotechnology, A. C. Tech Campus, Anna University, Chennai - 600 025, Tamil Nadu, India. ${ }^{2}$ Department of ECE, \\ K. L. University, Vaddeswaram - 522 502, Andhra Pradesh, India. Email: rengsahaa@gmail.com
}

Received: 18 October 2016, Revised and Accepted: 29 January 2017

\section{ABSTRACT}

Objective: Development of green nanotechnology is generating interest of researchers toward eco-friendly biosynthesis of nanoparticles (NPs). In this study, biosynthesis of stable copper (Cu) NPs was done using Passiflora foetida leaf extract.

Materials and Methods: First, we prepared leaf extract of Passiflora foetida in deionized water. This extract added to 20 mMol of Cupric sulfate solution, and we observed the change in color of the solution from colorless to colored solution; this indicates that there is a formation of CuNPs.

Results: These biosynthesized CuNPs were characterized with the help of ultraviolet visible spectroscopy, X-ray diffraction, and Fourier transform infrared spectroscopy. The spectroscopic analysis of synthesized CuNPs showed the maximum absorbance at $350 \mathrm{~nm}$ indicating the presence of biosynthesized CuNPs in the reaction mixture.

Conclusion: It was observed that the P. foetida leaf extract can reduce Cu ions into CuNPs within 8-10 minutes of reaction time. Thus, this method can be used for rapid and eco-friendly biosynthesis of stable CuNPs.

Keywords: Antimicrobial activity, Biosynthesis, Copper nanoparticles, Passiflora foetida.

(C) 2017 The Authors. Published by Innovare Academic Sciences Pvt Ltd. This is an open access article under the CC BY license (http://creativecommons. org/licenses/by/4. 0/) DOI: http://dx.doi.org/10.22159/ajpcr.2017.v10i4.15744

\section{INTRODUCTION}

In recent years, nanotechnology has attracted many researchers from various fields such as biotechnology, physics, chemistry, material sciences, engineering, and medicine. Nanoparticles (NPs) are synthesized by physical and chemical methods; these are suffering from drawbacks such as hazardous reaction condition, longer time, expensive reagent, tedious process to isolate NPs $[1,2]$. Hence, there is scope to develop new methods for the synthesis of NPs which should be required less drastic reaction condition, inexpensive reagent, and eco-friendly. In recent years, copper $(\mathrm{Cu}) \mathrm{NP}$ have attracted much attention of researchers because of its application in wound dressings and biocidal properties [3,4] potential industrial use such as gas sensors, catalytic process, high-temperature superconductors, and solar cells [5-7]. In literature, the CuNPs are synthesized from vapor deposition [8], electrochemical reduction [9], radiolysis reduction [10], thermal decomposition [11], chemical reduction of $\mathrm{Cu}$ metal salt [12], and room temperature synthesis using starch and hydrazine hydrate [13]. In recent, green synthesis of CuNPs was achieved using microorganisms [14], plant extract [15]. Passiflora foetida is commonly known as passion fruit. It is an exotic fast-growing perennial vine. The genus Passiflora belongs to Passifloraceae family that includes the passion fruit. Medicinal plants have played a significant role in a variety of ancient traditional systems of medicine. They are rich sources of bioactive compounds and thus serve as an important raw material for drug production and have become a target for the search of new drugs [16]. The plants are the invaluable, incredible, and traditional sources for the curability of various diseases in the form of medicines. Leaves of the plant utilized as folk medicine for treatment of antianxiety, stress, and insomnia. In addition, the plant has been used for its properties such as sedative, antiproliferative, antibacterial, antianxiety, leishmanicidal, antispasmodic, emetic, dressing for wounds, and antiulcer. The constituent of $P$. foetida is alkaloids, phenols, glycosides, flavonoids, cyanogenic compounds, passifloricins, polypeptides, and alpha-pyrones [17].

\section{MATERIALS AND METHODS}

Materials

All the reagents used in this experiment were obtained from Sigma-Aldrich Chemicals, India. Double-distilled water was utilized for this process. Filtration was established using Whatman No. 1 filter papers. Glasswares used for the complete reactions were washed well, rinsed with double-distilled water and dried in hot air oven.

\section{Collection of plant leaf}

P. foetida leaf was collected from Villupuram district. The collected leaf was tightly packed with a polyethene bag and then transfer to the laboratory. Then, it was washed with distilled water twice and kept under room temperature for 2 weeks in the dark condition. Then, it was made into powder using blender.

\section{Preparation of leaf extracts}

Plant leaf extract of $P$. foetida was prepared by first washing the leaves surface with running tap water followed by distilled water. The leaves were then dried in oven and crushed in powder form. To prepare the plant broth solution, $5 \mathrm{~g}$ powder of $P$. foetida leaves was taken in a $250 \mathrm{ml}$ beaker with $100 \mathrm{ml}$ of distilled water. Stirred vigorously and boiled the solution for 20 minutes at $80^{\circ} \mathrm{C}$. The extract was filtered through Whatman No. 1 filter paper. The filtrate thus obtained was stored in refrigerator for further experiments.

\section{Synthesis of CuNPs using $P$. foetida leaf extracts}

About $40 \mathrm{ml}$ of $P$. foetida aqueous leaf extract was added to $20 \mathrm{ml}$ of $20 \mathrm{mM}$ Cu sulfate solution in a $250 \mathrm{ml}$ flask under vigorous stirring. After 15 minutes, the green color of the solution was turned into reddish brown on keeping the reaction mixture in an oven at $80^{\circ} \mathrm{C}$ for $4 \mathrm{hrs}$. The appearance of reddish brown color indicated the complete reduction of $\mathrm{CuSO}_{4}$ solution and formation of CuNPs. The CuNPs thus obtained were purified by repeated centrifugation method at $9000 \mathrm{rpm}$ for 30 minutes followed by redispersion of the pellet in distilled water. The formation of CuNPs was strongly inferred by visual observation 
followed by ultraviolet-visible (UV-Vis) spectrum, Fourier transform infrared (FTIR), scanning electron microscope (SEM), X-ray diffraction (XRD), and energy dispersive analysis X-ray (EDAX) studies.

\section{Characterization of silver (Ag) NPs}

The synthesized CuNPs were characterized through UV-Vis spectrophotometer HITACHI U2300. The reduction of CuNPs was monitored by UV-spectrophotometer range of absorbance from 250 to $480 \mathrm{~nm}$. Synthesized AgNPs were characterized by FTIR to identify the biomolecules that were responsible for the reduction of AgNPs Jusco 5300 model with the wavelength range from 400 to $4000 \mathrm{~cm}^{-1}$ were used. SEM analysis was used to characterize surface morphology, using Supra Zeiss with a resolution of $1 \mathrm{~nm}$ at $30 \mathrm{kV}$ with $20 \mathrm{~mm}$ Oxford EDS detector. XRD studies were carried out to study the crystal structure of the synthesized AgNPs, the dried samples were coated on XRD grid, and the spectra were recorded using PANalytical X-pert X-ray generator operated at a voltage of $40 \mathrm{kV}$ and a current of $30 \mathrm{Ma}$.

\section{Antimicrobial activity}

Antibacterial activity of CuNPs synthesized from $P$. foetida aqueous leaf extract was determined by Agar well diffusion method. Muller and Hinton agar (MHA) medium (Hi-Media Pvt. Ltd., Mumbai) was used as a medium for this study [18]. The medium and other required glass wares were sterilized through autoclave process at $121^{\circ} \mathrm{C}$ for 15 minutes. After some time, MHA medium is poured into the petri plate. Following the solidification of medium, the inoculums were spread on the solid plates with sterile swab moistened with the bacterial suspension. The disc was placed in the wells made on MHA plates, and $20 \mu$ of sample (Concentration: 100, 75, 50 and $25 \mu \mathrm{g} / \mathrm{ml}$ ) were placed in the disc. The plates were incubated at $37^{\circ} \mathrm{C}$ for $24 \mathrm{hrs}$. Then, the antimicrobial activity was determined by measuring the diameter of zone of inhibition.

\section{RESULTS AND DISCUSSION}

\section{UV-analysis}

UV-Vis spectroscopy measures the extinction (scatter + absorption) of light passing through a sample. NPs have unique optical properties that are sensitive to the size, shape, concentration, agglomeration state, and refractive index near the NP surface, which makes UV-Vis a valuable tool for identifying, characterizing, and studying nanomaterials. The synthesized CuNPs were characterized through UV/Vis spectrophotometer Lambda 35. The biosynthesized CuNP was monitored by UV-spectrophotometer range of absorbance from 250 to $800 \mathrm{~nm}$. UV-Vis absorption spectrum shows peaks characteristics of the surface plasmon resonance of CuNPs [19]. The spectroscopic analysis of synthesized CuNPs showed the maximum absorbance at $350 \mathrm{~nm}$ indicating the presence of biosynthesized CuNPs in the reaction mixture. These experimental investigations were found to be in good agreement with the results already presented in the literature by Ashajyothi et al., 2014 (Fig. 1) [20].

\section{FTIR analysis}

FTIR measurements were done for both the aqueous fresh $P$. foetida leaf extract and the synthesized dried CuNPs to recognize the possible phytoconstituents responsible for the bioreduction, capping and efficient stabilization of the synthesized CuNPs. The FTIR spectra of the leaf extract and the synthesized CuNPs are shown in Fig. 2a and b. The spectrum was recorded in the wavelength region between 400 and $4000 \mathrm{~cm}^{-1}$.

The spectrum of aqueous fresh leaf extract (Fig. 2a) shows the peaks at wave numbers $3474,674 \mathrm{~cm}^{-1}$ and $1644,2361 \mathrm{~cm}^{-1}$. The peak at $1644 \mathrm{~cm}^{-1}$ was due to the presence of $\mathrm{C}=0$ stretching. The peak at $3474 \mathrm{~cm}^{-1}$ showed broad $\mathrm{O}-\mathrm{H}$ stretching of phenolic compound. The N-H stretching of primary amine was obtained from the peak at $674 \mathrm{~cm}^{-1}$. The alkyne stretching frequency was obtained at $2361 \mathrm{~cm}^{-1}$.

FTIR measurement of CuNPs showed the absorption peaks at 3412, 1625, 623, $2361 \mathrm{~cm}^{-1}$ in Fig. 2b. Peak at 1625 corresponds to $\mathrm{C}=0$ stretching of amides and 3412 corresponds to $\mathrm{O}-\mathrm{H}$ stretching of

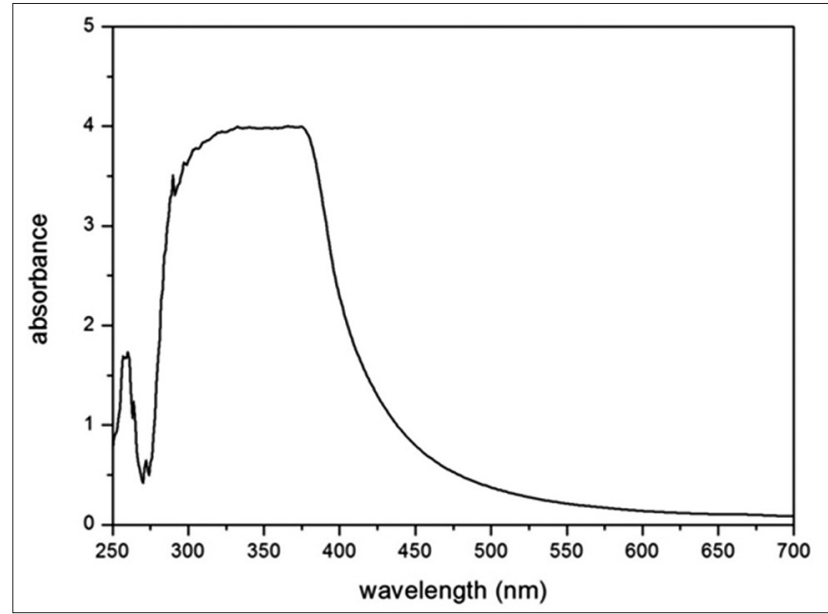

Fig. 1: Ultraviolet-visible spectrum of copper nanoparticles

phenolic compound. The other peaks obtained at 623 and $2361 \mathrm{~cm}^{-1}$ were due to $\mathrm{N}-\mathrm{H}$ stretching of primary amine and alkyne. On the basis of the peaks obtained for CuNPs, it is concluded that biosynthesized CuNPs might be surrounded by any one of these bioactive molecules such as polyphenols, alkaloids, and terpenoids which are in compliance with the already established facts in the literature [21].

The FTIR spectrum of the CuNPs indicates the lowering of peak intensity for $\mathrm{O}-\mathrm{H}$ stretch of phenolic compounds, confirming the reduction of CuNPs, which have been possibly proceeded via these groups. This indicates that water soluble compound such as polyphenols and terpenoids are present in extract. Therefore, it can be inferred that the biomolecules present in leaf extract, namely, flavonoids, alkaloids, and terpenoids might be responsible for the reduction of $\mathrm{Cu}$ ions to CuNPs due to their markable reducing capacity. Ulubelen et al. studied the species of $P$. foetida and reported the presence of C-glycosyl flavonoids such as chrysoeriol, apigenin, isovitexin, vitexin, 2"-xylosylvitexin, luteolin-7-B-D-glucoside, and kaempferol in enormous amount, which further supports our analysis for concluding flavonoids as one of the major reducing and capping agent (Fig. 2).

\section{SEM analysis}

The morphology of the biosynthesized dried CuNPs was examined by S-3400N SEM. The obtained SEM image shows that the product is mainly made of particle-like $\mathrm{Cu}$ nanoclusters with size ranges from 150 to $200 \mathrm{~nm}$. However, with high magnification, further observation reveals that these $\mathrm{Cu}$ nanoclusters are assembled by smaller NPs, which exhibit good uniformity, and the average diameter is about $24.54 \mathrm{~nm}$ (Fig. 3) [22].

\section{XRD analysis}

The crystal structure and size of the NPs are verified by XRD analysis. Peaks observed at $2 \theta$ values of $42.47,51.73$ and 73.42 correspond to (111), (200) and (220) planes of zero-valent CuNPs. These three peaks are quite consistent with those of the standard JCPDS Card No. 04-0836 for the standard spectrum of the pure face centered cubic (FCC) CuNPs (Fig. 4).

Besides the zero-valent CuNPs peaks, several other diffraction peaks appeared at 36.43, 61.58, 73.42 and 77.19 corresponding to (111), (220), (311) and (222) planes of cuprite NPs, respectively, indicate the formation of cubic $\mathrm{Cu}$ (I) oxide nanocrystals [23]. XRD peaks observed for cuprite NPs are matched well with the standard powder diffraction card of body centered cubic cuprite (JCPDS No. 05-667) [24]. The mean size of the crystalline zero-valent $\mathrm{Cu}$ and $\mathrm{Cu}$ oxide NPs calculated from the major diffractions peaks using the Scherrer formula is about 24.54 and $21.19 \mathrm{~nm}$, respectively.

Scherrer formula, $D=K \lambda / \beta \cos \theta$ 


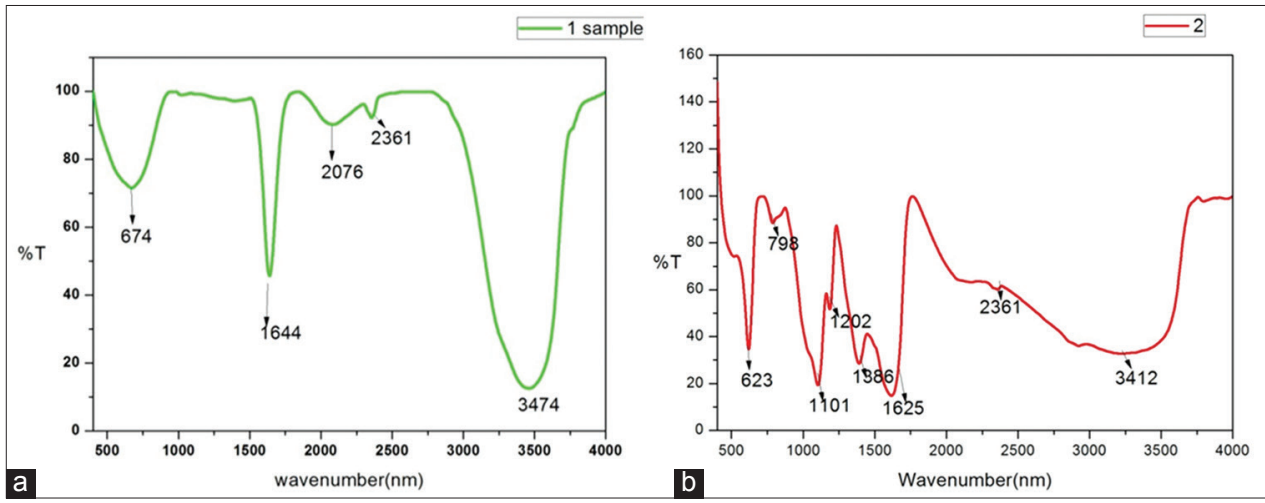

Fig. 2: (a) Fourier transform infrared (FTIR) spectrum of leaf extract, (b) FTIR spectrum of copper nanoparticles

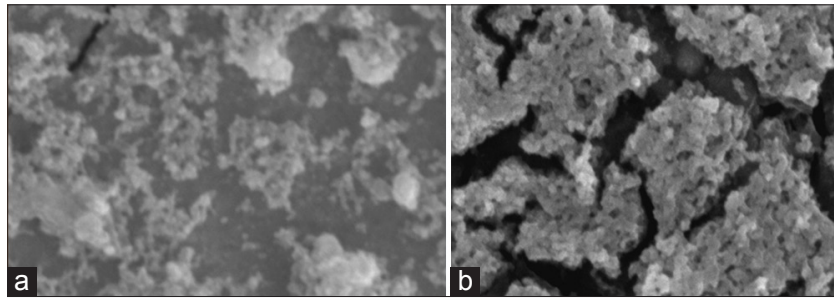

Fig. 3: (a) Scanning electron microscope image of biosynthesized copper nanoparticles, (b) high magnification

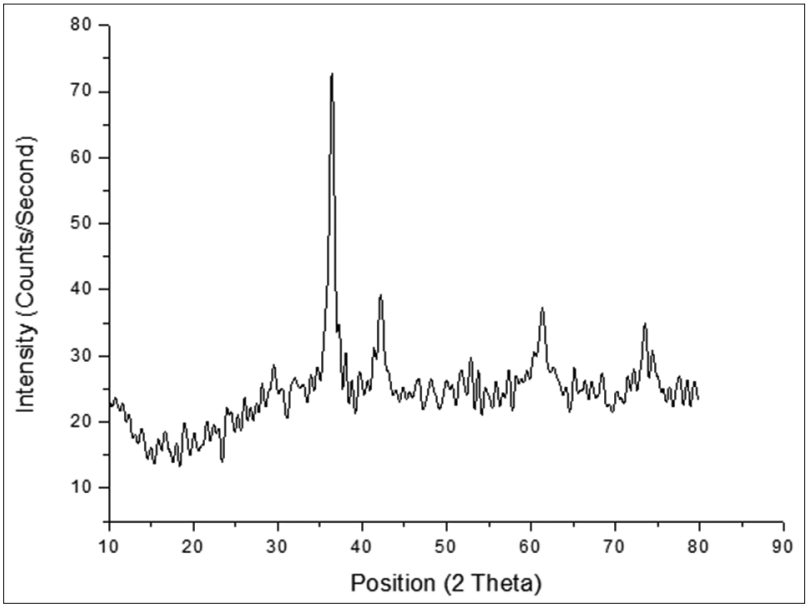

Fig. 4: X-ray diffraction pattern of biosynthesized copper nanoparticles at $2 \theta$

Where, $\mathrm{D}$ is the average size of crystallite, $\mathrm{K}$ is the Scherer constant with a value from 0.9 to $1, \lambda$ is the wavelength of the $X$-ray source $(0.1541 \mathrm{~nm})$ used in XRD, $\beta$ is the full width at half maximum of the diffraction peak, and $\theta$ is the Bragg's angle.

\section{EDAX analysis}

Energy-dispersive X-ray report confirms the elemental composition for the synthesized CuNPs.

This analysis also gave information about the weight percentage of CuNPs, depicting 49.95 weight $\%$ of $\mathrm{Cu}$ and 50.05 weight $\%$ of oxygen. As the analysis was done 1 month after the powder preparation, an aerial oxidation might have taken place which led the formation of $\mathrm{Cu}$ oxide (Fig. 5) [25].

\section{Dynamic light scattering}

The average size of the particles, size distribution, and polydispersity index (PDI) of the CuNPs was determined by particle size analyzer.
Table 1: Antimicrobial activity of biosynthesized CuNPs against pathogens at different concentrations

\begin{tabular}{lllll}
\hline Organisms & \multicolumn{4}{l}{ Zone of inhibition $(\mathbf{m m})$} \\
\cline { 2 - 5 } & \multicolumn{4}{l}{ Concentration $(\boldsymbol{\mu g} / \mathbf{m l})$} \\
\cline { 2 - 5 } & $\mathbf{1 0 0}$ & $\mathbf{7 5}$ & $\mathbf{5 0}$ & $\mathbf{2 5}$ \\
\hline E. coli & 15 & 13 & 10 & 6 \\
S. typhimurium & 14 & 12 & 9 & 6 \\
A. aceti & 10 & 7 & 5 & \\
\hline
\end{tabular}

E. coli: Escherichia coli, S. typhimurium: Salmonella typhimurium

A. aceti: Acetobacter aceti, CuNPs: Copper nanoparticles

Size distribution graph shows the average particle diameter is $318 \mathrm{~nm}$ and PDI is 0.200 . The average particle size and PDI revealed that the produced CuNPs are monodispersed (Fig. 6) [26].

\section{Antimicrobial activity of CuNPs}

Antimicrobial activity of biosynthesized CuNPs was studied against various pathogenic bacteria using agar well diffusion method. The test organisms used were Escherichia coli, Salmonella typhimurium, and Acetobacter aceti. Biosynthesized CuNPs showed clear zone of inhibition as indicated in Table 1. Maximum zone of inhibition $(15 \mathrm{~mm})$ was observed with $E$. coli, next was $S$. typhimurium with $14 \mathrm{~mm}$ zone of inhibition and A. aceti showed least zone of inhibition of $10 \mathrm{~mm}$ at $1000 \mu \mathrm{g} / \mathrm{ml}$ of CuNPs (Plate 1).

From the above result, it can be concluded that the zone of inhibition increases with increase in concentration of NPs. At the lower concentration of $250 \mu \mathrm{g} / \mathrm{ml}, A$. aceti was found to be resistant and at higher concentration showed the antimicrobial activity against Acetobacter. The occurrence of an inhibition zone clearly indicates the antimicrobial potential of CuNPs, disrupting the membrane. The reason could be that the smaller size of the CuNPs along with bound phytoconstituents, viz., flavonoids (chrysoeriol and apigenin), polyphenols and other biomolecules get tightly adsorbed on the surface of the bacterial cells. The presence of these bound phytoconstituents might be responsible for further enhancing the antimicrobial potential of biosynthesized CuNPs, leading to the disruption of bacterial membrane, thereby causing the leakage of intracellular components, thus killing the bacterial cells. Another proposed mechanism involves the association of $\mathrm{Cu}$ with oxygen and its reaction with sulfhydryl $(-\mathrm{S}-\mathrm{H})$ groups on the cell wall to form R-S-S-R bonds, thereby blocking respiration and causing cell death [27].

\section{CONCLUSION}

CuNPs were synthesized using aqueous leaf extract of $P$. foetida through green synthesis route. The leaf extract of $P$. foetida was found efficient for the synthesis of CuNPs. This method has merits over other reported methods such as easy availability of starting materials, inexpensive 


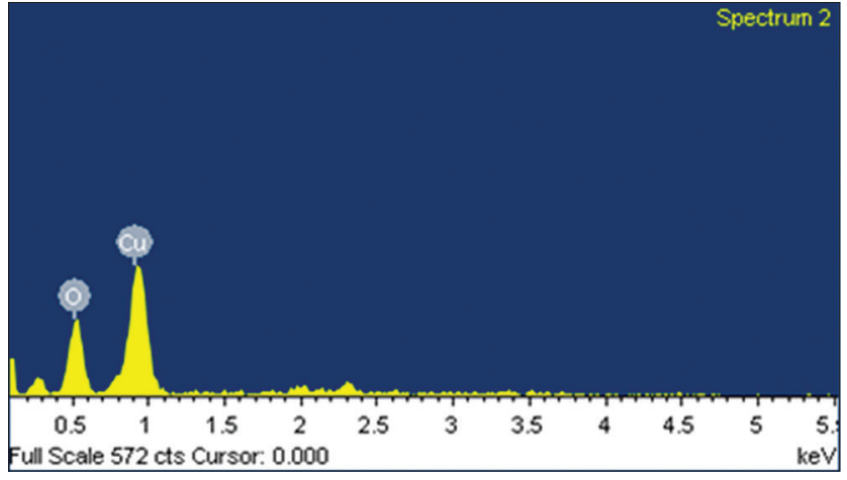

Fig. 5: Energy dispersive analysis X-ray analysis of copper nanoparticles

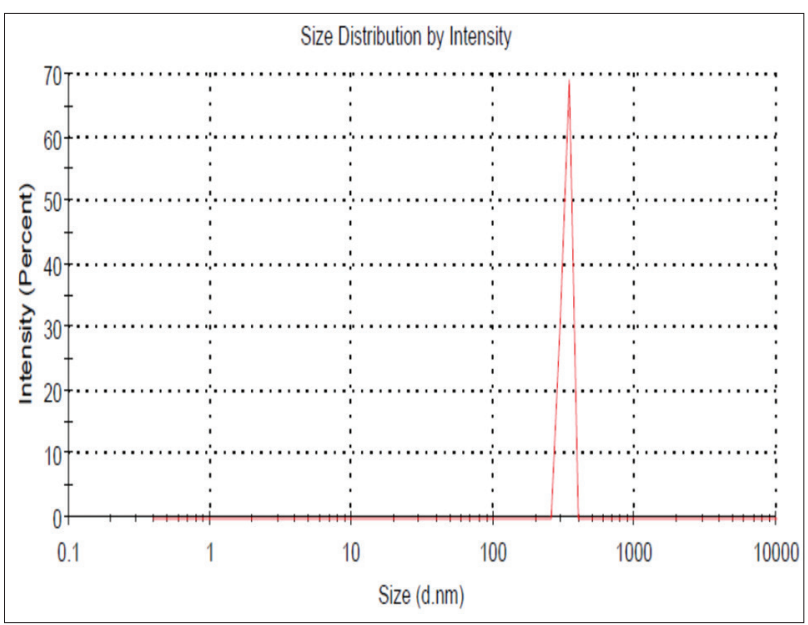

Fig. 6: Particle size distribution

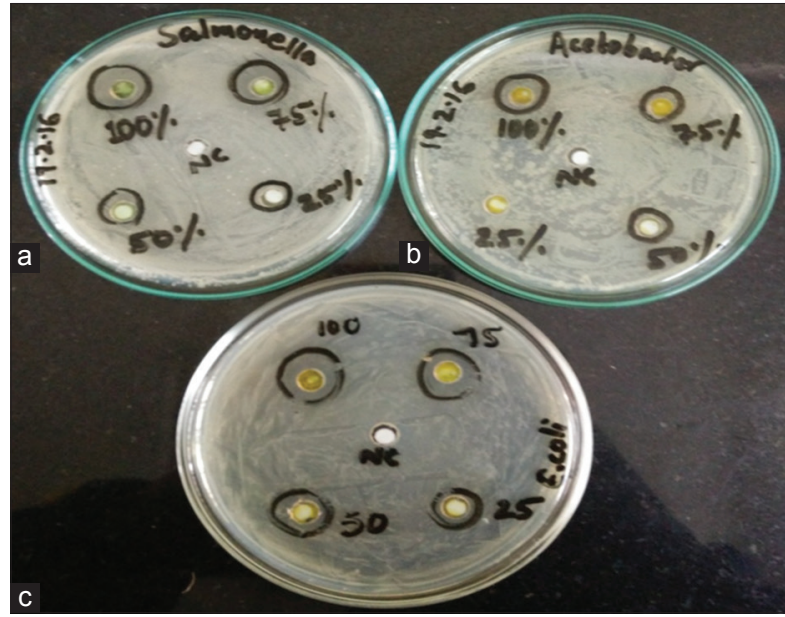

Plate 1: Diameter of zones of inhibition of (a) Escherichia coli,

(b) Salmonella typhimurium, and (c) Acetobacter aceti

process, ease of conduction at laboratory, simple reaction conditions, avoidance of use of expensive, hazardous and toxic reagents and pollution free. The synthesized CuNPs were characterized and assessed by UV-Vis spectrometer, FTIR spectroscopy, powdered XRD, SEM with EDAX (SEM with EDAX) and dynamic light Scattering. UV surface plasmon peak reveals the formation of CuNPs in the reaction mixture, which is further confirmed by EDAX analysis. FTIR interpretation gives an insight about the probable bioactive molecules which are acting as reducing and capping agents during the biosynthesis of CuNPs. XRD analysis affirms the crystalline nature (FCC) and mean size (24.54 nm) of CuNPs, which is in accordance with SEM result. XRD and EDAX result indicate the presence of $\mathrm{Cu}$ oxide NPs along with $\mathrm{Cu}$ zero valent NPs. The phenolic groups, for instance, chrysoeriol and kaempferol, present in fresh aqueous $P$. foetida leaf extract exhibit excellent antioxidant activity and these phenols can react with a free radical to form the phenoxyl radicals. Therefore, the use of natural anti-oxidant for the synthesis of CuNPs seems to be an effective root for the green synthesis. The biologically synthesized CuNPs shows excellent antibacterial activity in the reaction mixture.

\section{REFERENCES}

1. Lanje AS, Sharma SJ, Pode RB, Ningthoujam RS. Synthesis and optical characterization of copper oxide nanoparticles. Adv Appl Sci Res 2010;1(2):36-40

2. Yang G, Chai S, Xiong X, Zhang S, YU L, Zhang P. Preparation and tribological properties of surface modified $\mathrm{Cu}$ nanoparticles. Trans Nonferrous Met Soc China 2012;22(2):366-72.

3. Borkow G. Molecular mechanisms of enhanced wound healing by copper oxide impregnated dressings. Wound Repair Regen 2010;18(2):266-75.

4. Borkow G, Zatcoff RC, Gabbay J. Reducing the risk of skin pathologies in diabetics by using copper impregnated socks. Med Hypotheses 2009;73(6):883-6

5. Li Y, Liang J, Tao Z, Chen J. CuO particles and plates: Synthesis and gas-sensor application. Mater Res Bull 2008;43(8-9):2380-5.

6. Carnes LC, Klabunde KJ. The catalytic methanol synthesis over nanoparticle metal oxide catalysts. J Mol Catal A Chem 2003;194(1-2):227-36.

7. Guo Z, Liang X, Pereira T, Scaffaro R, Hahn HT. CuO nanoparticle filled vinyl-ester resin nanocomposites: Fabrication, characterization and property analysis. Compos Sci Technol 2007;67(10):2036-44.

8. Choi H, Park SH. Seedless growth of free-standing copper nanowires by chemical vapor deposition. J Am Chem Soc 2004;126:6248-9.

9. Huang L, Jiang H, Zhang J, Zhang Z, Zhang P. Synthesis of copper nanoparticles containing diamond like carbon films by electrochemical method. Electrochem Commun 2006;8(2):262-6.

10. Joshi SS, Patil SF, Iyer V, Mahumuni S. Radiation induced synthesis and characterization of copper nanoparticles. Nanostruct Mater 1998:10(7):1135-44.

11. Aruldhas N, Raj CP, Gedanken A. Synthesis, characterization, and properties of metallic copper nanoparticles. Chem Mater 1998;10(5):1446-52.

12. Hashemipour H, Rahimi ME, Pourakbari R, Rahimi P. Investigation on synthesis and size control of copper nanoparticle via electrochemical and chemical reduction method. Int J Phys Sci 2011;6(18):4331-6.

13. Surmawar NV, Thakare SR, Khaty NT. One-pot, single step green synthesis of copper nanoparticles: SPR nanoparticles. Int J Green Nanotechnol 2011;3(4):302-8.

14. Honary S, Barabadi H, Gharaeifathabad E, Naghibi F. Green synthesis of copper oxide nanoparticles using Penicillium aurantiogriseum, Penicillium citrinum and Penicillium waksmanii. Dig J Nanomater Biostruct 2012;7(3):999-1005

15. Gunalan S, Sivaraj R, Venkatesh R. Aloe barbadensis miller mediated green synthesis of monodisperse copper oxide nanoparticles: Optical properties. Spectrochim Acta A Mol Biomol Spectrosc 2012;97:1140-4.

16. Aseervatham J, Palanivelu S, Panchanadham S. Semecarpus anacardium (Bhallataka) alters the glucose metabolism and energy production in diabetic rats. Evid Based complement Alternat Med 2011;10(4):1-9.

17. Sasikala V, Saravana S Parimelazhagan T. Evaluation of antioxidant potential of different parts of wild edible plant Passiflora foetida L. J Appl Pharm Sci 2011;1(4):89-96.

18. Bauer AW, Kirby WM, Sherris JC, Turck M. Antibiotic susceptibility testing by a standardized single disk method. Am J Clin Pathol 1966;45(4):493-6.

19. Zhu H, Zhang C, Yin Y. Nanotechnology 2005;16:3070.

20. Ashajyothi C, Kudsi J, Kelmani CR. Biosynthesis and characterization of copper nanoparticles from Enterococcus faecalis. Int J Pharm Bio Sci 2014;5(4):204-11.

21. Saranyaadevi K, Subha V, Ravindran RS, Renganathan S. Green synthesis and characterization of silver nanoparticle using leaf extract of Capparis zeylanica. Asian J Clin Res 2014;7(2):44-8.

22. Angrasan JK, Subbaiya R. Biosynthesis of copper nanoparticle by Vitis 
vinifera leaf aqueous extract and its antibacterial activity. Int J Curr Microbiol Appl Sci 2014;3(9):768-74.

23. Martis P, Fonseca A, Mekhalif Z, Delhalle J. Optimization of cuprous oxide nanocrystals deposition on multiwalled carbon nanotubes. J Nanopart Res 2010;12:439-48.

24. Waseda Y, Matsubara E, Shinoda K. X-ray Diffraction Crystallography: Introduction, Examples and Solved Problems. Berlin: Springer; 2011.

25. Zain NM, Stapley AG, Shama G. Green synthesis of silver and copper nanoparticles using ascorbic acid and chitosan for antimicrobial applications. Carbohydr Polym 2014;112:195-202.

26. Pavani KV, Srujana N, Preethi G, Swati T. Synthesis of copper nanoparticles by Aspergillus species. Lett Appl Nanobioscience 2013;2(2):110-3

27. Gopinath M, Subbaiya R, Selvam MM, Suresh D. Synthesis of copper nanoparticles from Nerium oleander leaf aqueous extract and its antibacterial activity. Int J Curr Microbiol Appl Sci 2014;3(9):814-8. 